\title{
Biomechanical study on the changes of stress in temporomandibular joints after the orthognathic surgery in patients with mandibular prognathism: a 3D finite element study
}

\author{
JINGHENG SHU ${ }^{1 \#}$, QUANYI WANG ${ }^{2,3 \#}$, HEDI MA ${ }^{1}$, HAIDONG TENG ${ }^{1}$, TINGHUI ZHENG ${ }^{1}$, ZHAN LIU $^{1 *}$ \\ ${ }^{1}$ Key Lab for Biomechanical Engineering of Sichuan Province, Sichuan University, Chengdu, China. \\ ${ }^{2}$ Key Laboratory of Deep Earth Science and Engineering, Ministry of Education, Sichuan University, Chengdu, China. \\ ${ }^{3}$ School of Aeronautics and Astronautics, Sichuan University, Chengdu, China. \\ \# The authors contributed equally to this study.
}

\begin{abstract}
Purpose: This study aimed to analyze the changes of the stress distributions in TMJs for the pre- and postoperative patients with mandibular prognathism under unilateral occlusions, a frequent occlusion in mastication. Methods: Pre- and six-mouth postoperative cone-beam computed tomography images of thirteen patients diagnosed with mandibular prognathism were scanned and used to construct complete maxillofacial models, assigned as the Pre and Post group, respectively. Another ten asymptomatic individuals were defined as the Control group. The inhomogeneous properties were assigned to the models. The muscle forces and boundary conditions corresponding to left and right unilateral occlusions were applied on the models. The analysis of variation (ANOVA) was chosen for the comparison among the groups. Results: The results showed that the Pre group had abnormal stress distributions ang higher stress level in TMJs, compared with those of the Post and Control groups. Moreover, from clinical cases, symptoms of temporomandibular disorders (TMDs) always followed with increased stresses. Conclusion: Generally, orthognathic surgeries could improve the stress distribution in TMJs of the patients with mandibular prognathism under the unilateral occlusions. However, the postoperative complications, especially symptoms of TMD, were closely related to changes of stress for patients with mandibular prognathism after orthognathic surgeries. Individual virtual surgery and finite element analysis should be conducted to prevent complications in TMJ.
\end{abstract}

Key words: temporomandibular joint (TMJ), finite element method (FEM), mandibular prognathism, temporomandibular disorders (TMD)

\section{Introduction}

The mandibular prognathism refers to the geometry of the mandible with lower dentition covering upper dentition, with a $25.5 \%$ prevalence in all kinds of mandibular deformities [27]. It can lead to chewing dysfunction, disorder occlusions and bad appearance, as well as psychological disturbances such as inferiority [2]. Moreover, symptoms of temporomandibular disorders (TMDs) also puzzle these patients, which is mainly caused by changes of internal degeneration of structures, such as disc displacement, condylar resorption, and disc perforation, etc. [26]. Bilateral sagittal split ramus osteotomy (BSSRO) is a surgery extensively used to correct mandibular prognathism with its good therapeutic effects on appearance and occlusion (Fig. 1). However, the consideration of structural changes of TMJ is limit. As a result, BSSRO brings some postoperative symptoms in TMJs; the most rele-

\footnotetext{
* Corresponding author: Zhan Liu, Key Lab for Biomechanical Engineering of Sichuan Province, Sichuan University, Chengdu, China, email address: bmeliuzhan@163.com

Received: March 20th, 2020

Accepted for publication: April 21st, 2020
} 

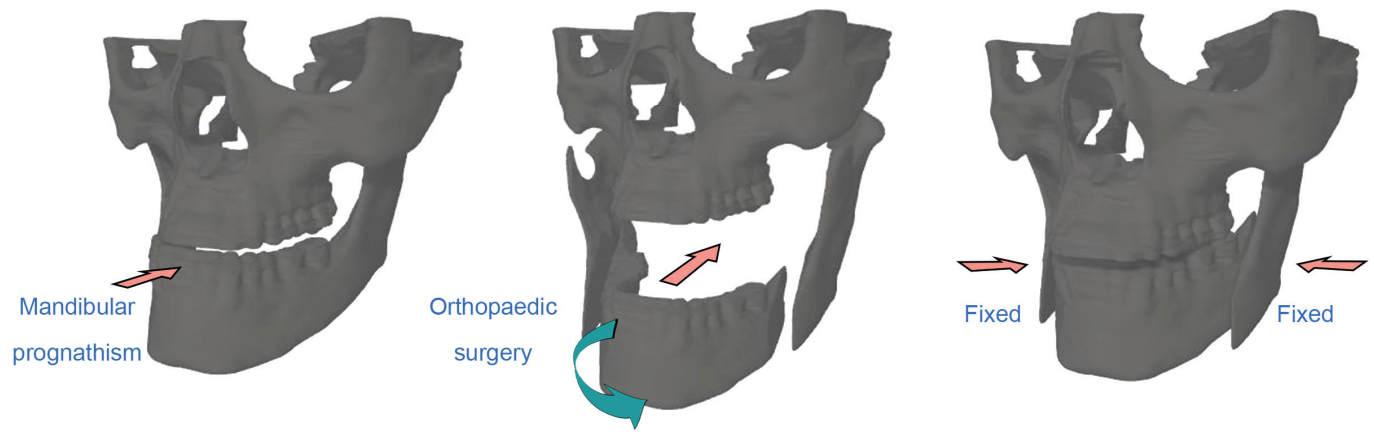

Fig. 1. Brief process of orthognathic surgeries for mandibular prognathism patients

vant symptoms is TMD [19], [26]. Previous studies reported that symptoms of TMDs have a great relationship with changes of the stresses in the TMJ [10], [24]. Thus, it is necessary to understand the relationships between mandibular prognathism, BSSRO, and stress distributions in TMJ.

BSSRO affects functions of TMJ in different ways. Some researchers pointed out that although BSSRO beautifies the appearance of patients, it still has possibility causing TMD symptoms [26]. And the positional and morphological changes of TMJ after surgeries may contribute to TMDs [1], [7]. Moreover, different surgical fixations also affect masticatory forces and TMJ loads after BSSRO through the animals' experiments, furtherly leading to TMDs [14], [15]. However, opposite opinions indicated that BSSRO restores occlusions for the patients, and alleviates or eliminates the symptoms of TMD [6], [21]. From biomechanical point of view, a proper biomechanical environment in TMJs has a good effect of recovery. Contrarily, various symptoms of TMD are associated with increased loads in TMJs [10]. Thus, the stress distributions in TMJs before and after BSSRO for the patients with mandibular prognathism should be investigated to find the relationship of postoperative TMD and loads.

Previous studies concentrated on morphological and positional changes of TMJs under the central occlusion [6], [25]. However, as unilateral occlusions are often seen in mastication, it is more meaningful to simulate the internal stress distribution under unilateral occlusions. This study firstly aims to evaluate differences of stress distribution in TMJs between pre- and postoperative mandibular prognathism patients, then to compare to those of the asymptomatic subjects under unilateral occlusions. Furthermore, stress distributions in TMJs for patients with pre- and postoperative symptoms of TMD are also analyzed.

\section{Materials and methods}

\subsection{Establishment of the models}

Thirteen mandibular prognathism patients ( 7 women and 6 men, $23.00 \pm 2.98$ years old) before and after BSSRO were recruited and assigned as the Pre and Post groups, respectively. Another 10 asymptomatic volunteers ( 4 women and 6 men, $26.70 \pm 4.80$ years old) without TMJ diseases assigned as the Control group. The inclusion criteria for patients in our study were: exceeding 18 years old; without prior TMJ procedures; diagnosis of mandibular prognathism with their upper dentitions covered by lower ones. The inclusion criteria for asymptomatic subjects were mentioned in previous study [18]. The research protocol was approved by the Institutional Review Board. All subjects were provided written informed consent.

All these subjects were scanned by cone-beam computed tomography (CBCT) with total 290 to 340 image slices and a 0.4-mm slice thickness (Fig. 2A). Then, the models of mandible, maxilla, and disc for each subject were established according to CBCT images in Mimics 15.0 (Materialise, Leuven, Belgium) (Fig. 2B-C) [21]. Then, the 3D models including mandible, maxilla, and discs were imported into a finite element analysis software ABAQUS 6.13 (Dassault, SIMULIA, RI).

\subsection{Material properties}

Based on a previous research [9], the interaction among discs, condyle and temporal bone was considered as contact with a frictional coefficient 0.001 . Bone was considered as inhomogeneous according to empirical relations between gray values and elastic modulus [18] ((1)-(2)). The Poisson's ratios of articular discs and bone were 0.4 and 0.3 , respectively [22]. Accord- 


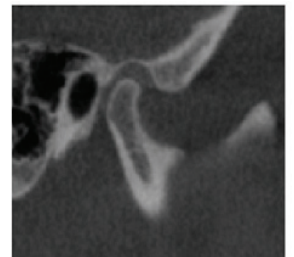

A

Asymptomatic subject

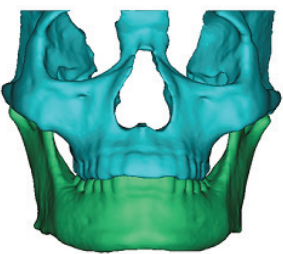

B
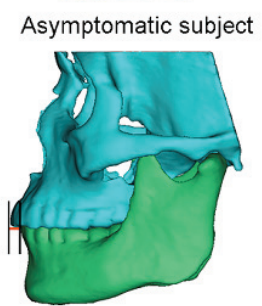

C

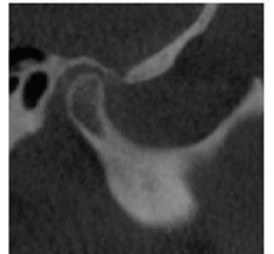

Preoperative pateint

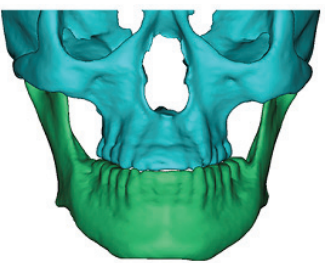

Preoperative pateint

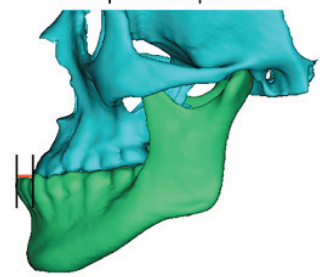

Preoperative pateint

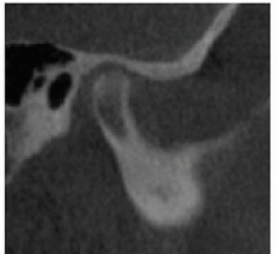

CT slice

Postoperative pateint

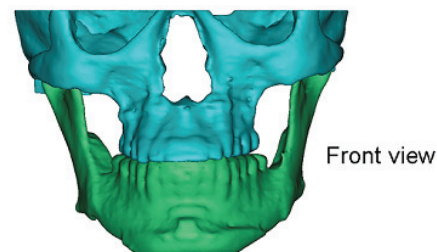

Postoperative pateint

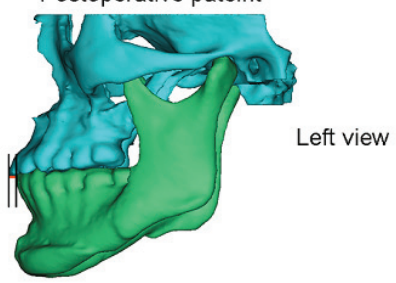

Postoperative pateint

Fig. 2. A-C CT and 3D models of an asymptomatic subject and patient before and after the surgery:

A - CT slices of TMJ for an asymptomatic subject, a patient before and after BSSRO;

B - 3D models of an asymptomatic subject, a patient before and after BSSRO (Front view);

C -3 D models of an asymptomatic subject, a patient before and after BSSRO (Left view)

ing to the formulas, units were transferred to corresponding units in ABAQUS.

$$
\text { Density }=-13.4+1017 \times \text { Gray value } \ldots
$$

Elastic modulus $=-388.8+5925 \times$ Density $\ldots$

\subsection{Mesh}

The models were meshed by the tetrahedron elements due to the complexity of the geometry. For the higher precision and contact algorithm, the mesh of TMJ regions was the 10-node quadratic element (C3D10M) (Fig. 3). The other regions were meshed by the 4-node linear elements (C3D4). The FE models were all consisted of over 150000 elements in total through previous convergence test [9].

\subsection{Loading and boundary conditions}

Muscle forces of unilateral occlusion were loaded on models [8], [16]. Apart from the central occlusion,

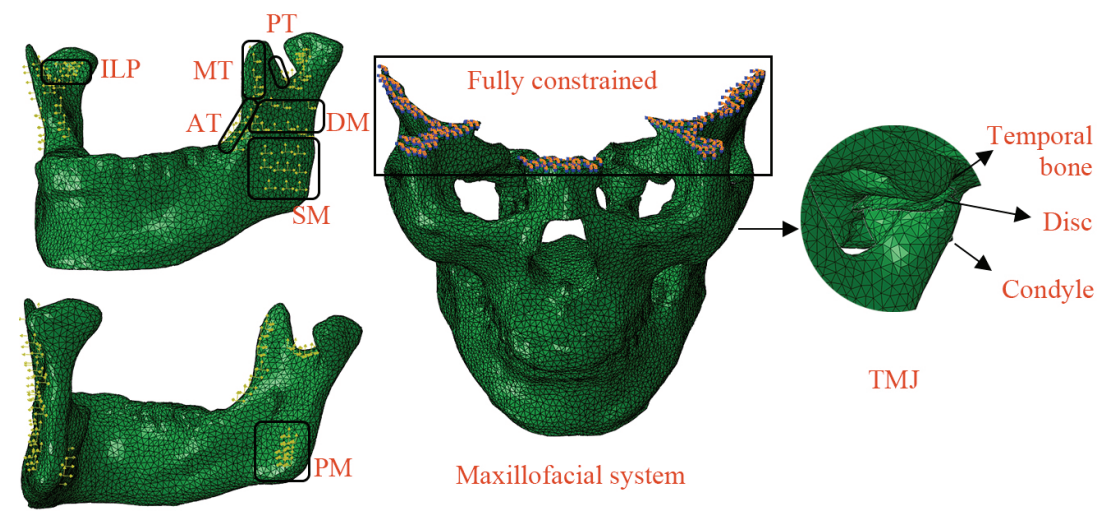

Fig. 3. The mesh, loadings and boundary conditions of the FE model with a detailed TMJ. Notes: SM - superficial masseter; DM - deep masseter; MP - medial pterygoid; AT - anterior temporalis; MT - middle temporalis; PT - posterior temporalis; ILP - inferior lateral pterygoid 
muscles of each side during unilateral occlusion had different efficiencies. Furthermore, each muscle force in this study was applied on 10 nodes in the muscle area to properly simulate the attached area of the muscles. The top surface of maxilla was fully constrained. Left unilateral molar clenching (LMOL) and right unilateral molar clenching (RMOL), meaning that unilateral clenching primarily by left or right molars respectively, were applied on all subjects. The ipsilateral side to clenching side defined as the working side, and the contralateral side to clenching defined as the non-working side. Thus, the left and right sides were working and non-working sides under the LMOL, and non-working and working sides under the RMOL, respectively. The attachments were added between bone structures and discs according to the previous study [9]. Maximum and minimum principle stresses were chosen for representing the stress level of TMJ, and von Mises stresses were used for the illustration of stress distributions in TMJs.

\subsection{Statistical analysis}

Maximum and minimum principle stresses for three groups were included in statistical analysis, and the analysis of variance (ANOVA) and Student $t$-tests were performed in SPSS 20.0 (SPSS Inc, Chicago, IL). The statistically analysis about comparisons of stresses at working and non-working sides among three groups was analyzed by paired-samples $t$-test. Statistical differences among three groups were analyzed by ANOVA. Since structures and functions of asymptomatic subjects were healthy and stress distributions of the working and non-working sides under both occlusions were similar, only right unilateral occlusion (RMOL) was shown and compared in results of the Control group. The significance of the analysis was achieved as $P<0.05$.

\section{Results}

\subsection{Stress comparisons between two sides}

Maximum and minimum principal stresses stresses for the Control group at the non-working side were significantly greater than those at the working side (Fig. 4). Postoperative maximum and minimum principle stresses at condyles exhibited significant differences between working and non-working sides, so as maximum principal stresses at temporal bones. However, no significant differences existed between both discs, condyles, and temporal bones in the Pre group.

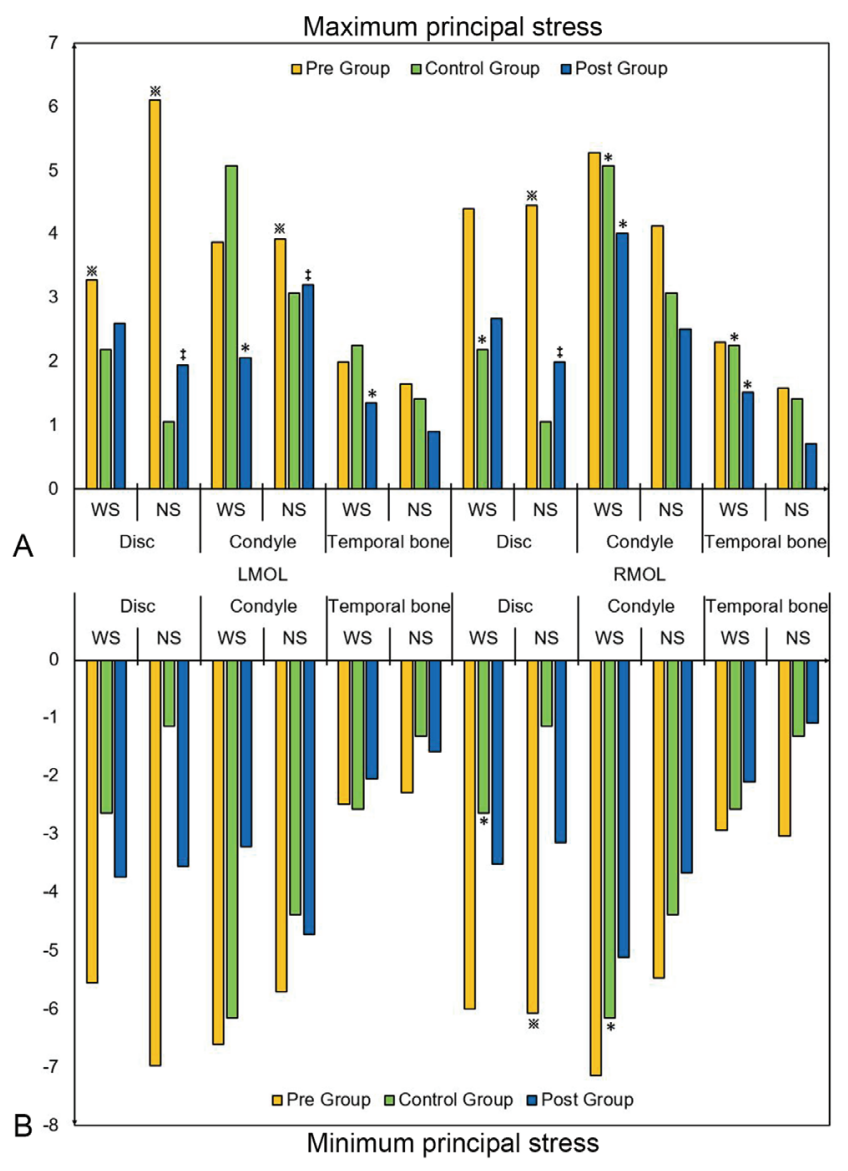

Fig. 4. A-B Comparisons of the peak stresses in the TMJs under the unilateral occlusions among the three groups (MPa) and stress comparisons of TMJs

between working and non-working sides (MPa). A - maximum principal stress; B: minimum principal stress; ※ exhibited significance between the Pre and Control group; $\$$ exhibited significance between the Post and Control group; * exhibited significance between two sides.

No significant difference existed between the Pre and Post group. LMOL - left unilateral molar clenching; RMOL - right unilateral molar clenching; WS - working side; NS - non-working side

\subsection{Changes of stress level}

Maximum principal stresses of TMJs in the Pre group were greater than those of the Control group, except for those of condyles and temporal bones at working sides under the left unilateral occlusion (Fig. 3). Moreover, stresses of the Control group were greater than those of the Post group, especially in the condyle and temporal bone. Significances 
among the three groups were concentrated at the left unilateral occlusion.

The trend of minimum principal stresses was similar to that of maximum principal stresses. Stress levels of the Post group dropped down and were lower than those of the Control group, especially in condyles and temporal bones (Fig. 4). Stress levels of discs in the Post group were lower than those in the Pre and Control groups.

\subsection{Changes of the stress distributions}

High stress regions of asymptomatic individuals were located at the anterior of temporal bone at the non-working side, while the stresses at the working side was uniform without high stress region, so as the stress of disc. However, the stresses of the Pre group became irregular (Fig. 5) (only 1 patient as an example). Five patients concentrated on the lateral side of temporal bone and disc at non-working side, other three concentrated on the posterior part of disc at nonworking side and another one concentrated on disc of the working sides. The remaining patients were close to normal. After the surgery, stress distributions for some patients were still abnormal and irregular, but the magnitudes was close to the Control group in this study.

\section{Discussion}

BSSRO provides an effective correction to remedy mandibular deformities. Although it has better performance on the improvement of appearance by changing bony structures, effects on TMJs are still divided. The remodeling of hard and soft tissues is also affected by orthognathic surgery [3], accompanied with changes of structures after the surgery. A previous study indicated that BSSRO provides a good improvement for facial asymmetric patients on stress distributions under the central and anterior occlusions, as well as recoveries of TMD symptoms [21]. There are also some opposite opinions for BSSRO, such as postoperative symptoms of TMD [20]. Furthermore, the unilateral occlusion is used with a higher frequency in daily mastication [23], providing a competitive influence on TMJ compared with central and anterior occlusions. Moreover, the stress analysis of mandibular prognathism patients under the unilateral occlusions is still lacking. Thus, the purpose of this study was to evaluate comparisons of the stress in TMJs under the unilateral occlusions between preand postoperative patients with mandibular prognathism.

The interaction of TMJs was considered as contact with a frictional coefficient 0.001 [11]. Five 3D maxillofacial models, whose material properties were

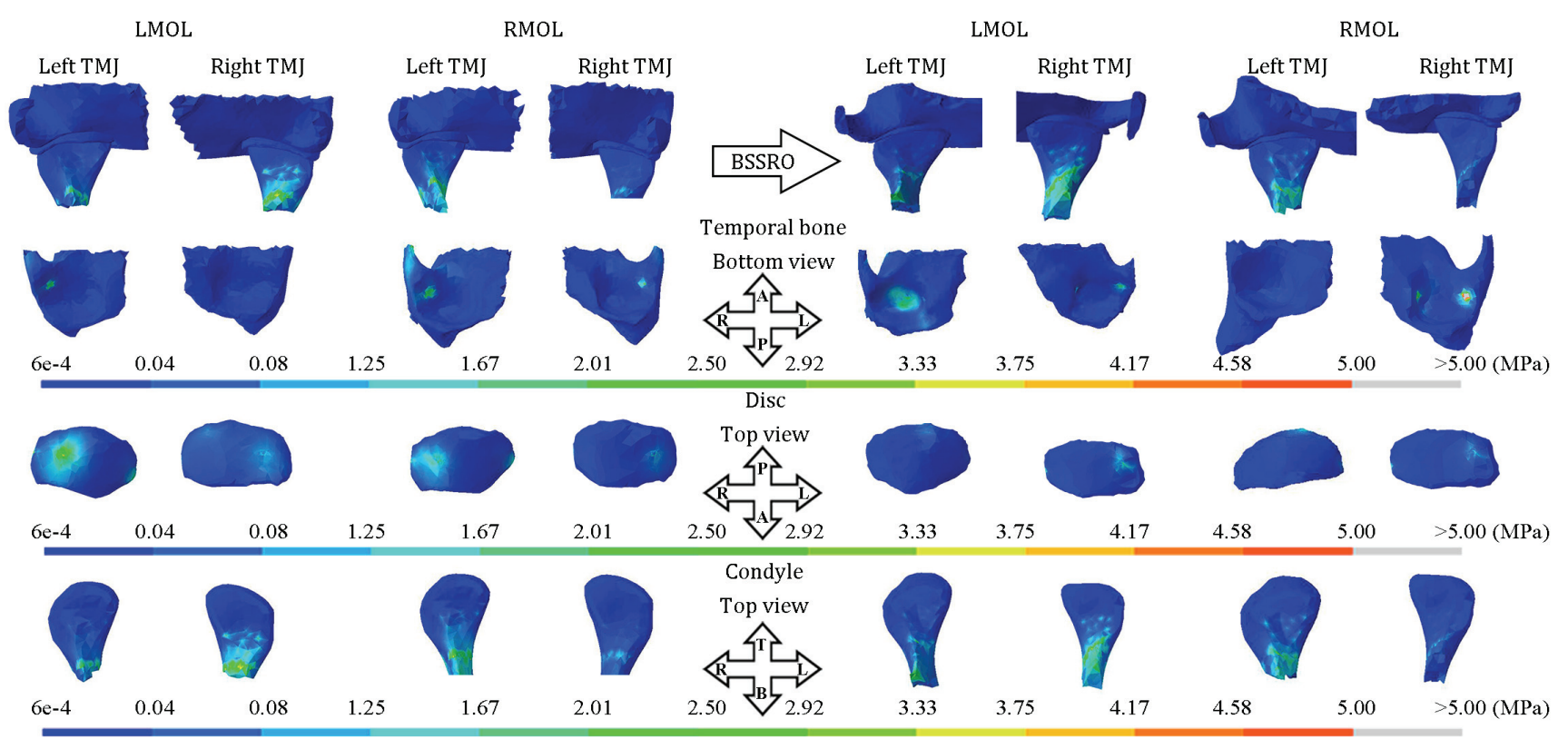

Fig. 5. The von Mises stress distributions of a mandibular prognathism patient before and after BSSRO.

LMOL - left unilateral molar occlusion, RMOL - right unilateral molar occlusion,

$\mathrm{A}$ - anterior, $\mathrm{P}$ - posterior, $\mathrm{L}$ - left, $\mathrm{R}$ - right, $\mathrm{T}$ - top, $\mathrm{B}$ - bottom 
similar to maxillofacial system, were used to validate this method [28]. To the 3D models, vertical pressure on the top surface of the maxilla was added to simulate central occlusion. Finite element models and 3D models shared the same geometries, material properties, loads, and boundary conditions. The strain rosettes were attached on the mandible to record the vertical and horizontal strains. The results showed that the differences between experimental and simulated strains of the same monitoring point were within $5 \%$. Thus, the interaction of TMJ simulated as contact with a 0.001 friction coefficient was reasonable. Moreover, the mandible and maxilla were considered as inhomogeneous materials, more realistic than homogeneous materials and rigid bodies.

\subsection{The appearance of patients after BSSRO}

The appearance of patients had a great improvement after surgeries. The position of condyles in mandibular prognathism patients was always nearer to the articular eminence, compared to the asymptomatic individuals (Fig. 2A), according to with da Silva et al.'s study [3]. The lower space would lead to the decrease of degree of freedom [29], [30], interfered with movements of the condyle in the glenoid fossa. In the case reports of this study, there was one patient that had serious preoperative limited opening of mouth. After surgeries, relative positions between upper and lower dentitions became normal with the recovery of limited opening of mouth (Fig. 2C). Thus, the proper interference in TMJs should be considered before the surgery to retain enough postoperative space between condyle and articular eminence, especially for patients with limited opening of mouth.

\subsection{Effect of the mandibular prognathism and BSSRO on stress comparisons between both sides}

The mandibular prognathism contributes to abnormal stress distributions in TMJs. Maximum and minimum principal stresses at the working side of the Control group were significantly lower than those at the non-working side. TMJs at working sides had more contact, while TMJs at non-working sides manifested greater stress level owing to larger movements at these sides under the unilateral occlusions. However, comparisons of stress between working and nonworking sides of postoperative patients were not sig- nificantly different no matter whether under the left or right unilateral occlusions (Fig. 4). The stress levels of bilateral TMJs became close, different from the normal status in healthy individuals. The structures at the working side were as unstable and loose as those at the non-working side under unilateral occlusions, causing larger bilateral movements. After surgeries, although significances between the both sides reappeared and the trend was approaching to the normal individuals, it was still far from the status of normal individuals.

\subsection{Effect of mandibular prognathism and BSSRO on stress level}

Maximum and minimum principal stresses stand for tensile and compressive characteristics, respectively. In this study, maximum and minimum principal stresses of the disc, condyle, and temporal bone of the Post group were obviously lower than those of the Pre group under both unilateral occlusions (Fig. 4). Moreover, maximum and minimum principal stresses for the Post group of condyle and temporal bone under the right unilateral occlusion were lower than those of the Control group. It meant that the right unilateral occlusion was positive for the patients' recovery to avoid high stress. These differences between capabilities of left and right unilateral occlusions may result from the right chewing side preference. Diernberger et al.'s study exhibited larger percentage of population preferred to use right chewing [5]. Long-time chewing side preference caused functional decline of masticatory and occlusal force of non-working sides [12], corresponding to the masticatory capability of left sides in this study. However, disadvantages of long-time chewing side preference appeared as changes of aesthetics and caused of facial asymmetry [12]. Although the right unilateral molar clenching was better for decreasing the stress six months after surgeries, it was still risky for occurrence of facial or muscular asymmetry, even the asymmetry of body [13], [17]. Thus, suggestions should be arisen to avoid chewing side preference from the beginning of postoperative recoveries.

\subsection{Effect of mandibular prognathism and surgeries on stress distributions}

As an asymmetric loading, the unilateral molar clenching not only produced an asymmetric stress discrepancy between both sides, but also changed the stress distributions and high stress regions. In the asymptomatic subjects, the high stress regions of the 
discs at the non-working side were located at the anterior band, consistent with the other asymmetric loadings [4]. However, the nature of the stress in discs was correspondingly changed with the structural changes in the patients. The lateral part and the posterior band of the disc became other high stress regions, which may lead to the lateral disc displacement if long-term unilateral molar clenching remained. After the orthognathic surgery, although the magnitude of the stress dropped down, some patients were still in an abnormal stress distribution. From that point, postoperative orthodontics and occlusal reconstruction should be involved as the subsequent treatment.

\subsection{Effect of mandibular prognathism and BSSRO on the symptoms of TMDs}

From the biomechanical standpoint, the extra stress could cause TMD [10]. In other words, increased stresses of preoperative patients changed the normal biomechanical environment in TMJs, would be followed by

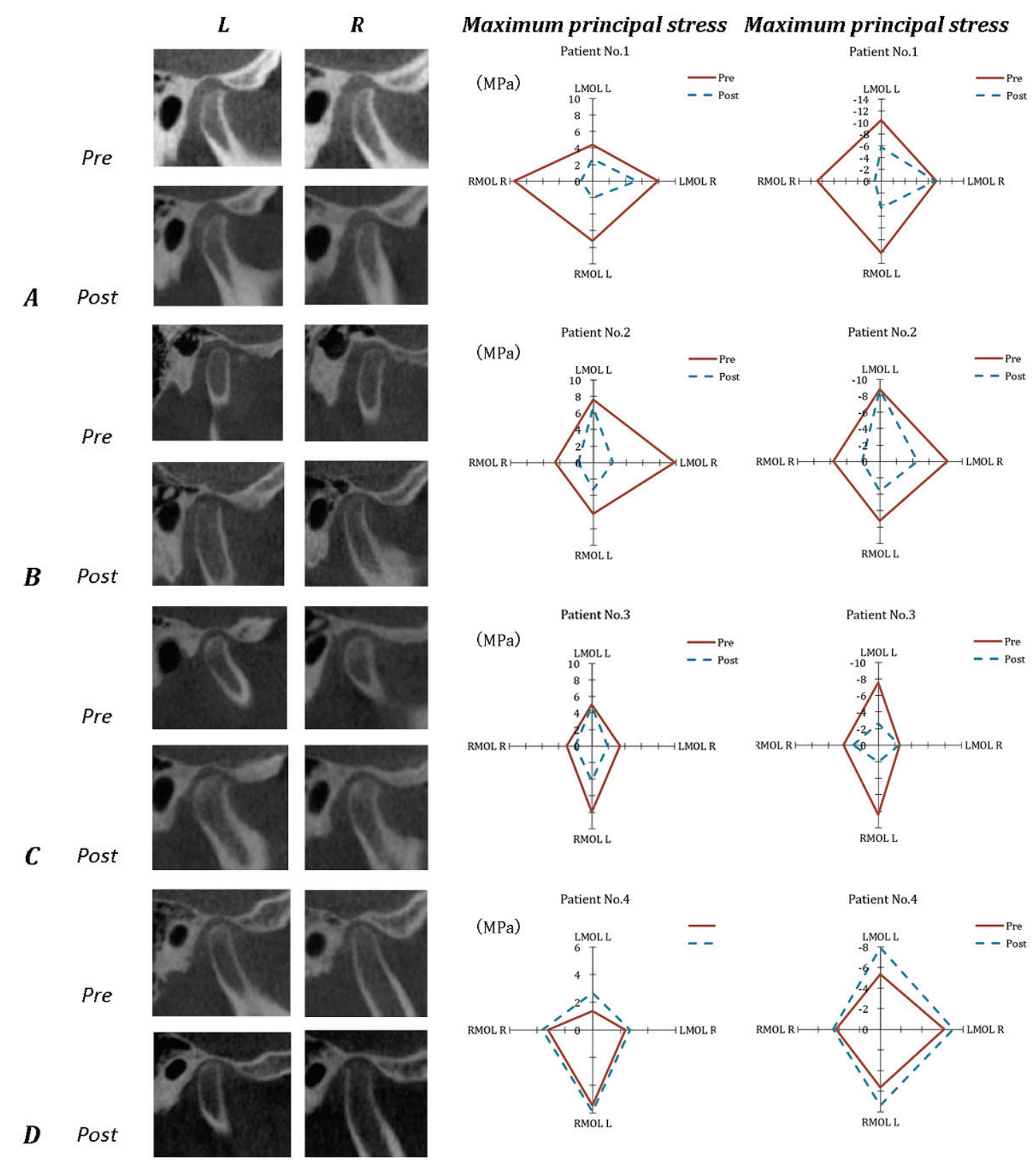

Fig. 6. The CT of TMJ and the comparisons of stress in disc between pre and postoperative patients associated with clicking joint. A - Patient No. 1 with preoperative clicking at right TMJ and without postoperative TMD, B - Patient No. 2 with preoperative clicking at left TMJ and with postoperative clicking only at beginning and ending of left TMJ during maximal opening. C - Patient No. 3 with preoperative clicking at both sides and without postoperative TMD. D - Patient No. 4 without preoperative TMD and with postoperative clicking at right TMJ 
TMD. In our study, stresses of two of three patients with joint clicking were disappeared after surgeries (Fig. 6A and $6 \mathrm{C}$ ), and symptoms of TMD in the remaining patient was alleviated (Fig. 6B). All the three patients were with the decreased stresses, always accompanied by the increased space between the anterior of condyle and articular eminence (Fig. 6). However, postoperative clicking of a patient without preoperative TMD appeared along with the increase of postoperative stresses (Fig. 6D). Thus, the occurrence of TMD in patients would originate from high stresses in the TMJ, consistently with previous study [10]. However, the stress level had individual differences. Although postoperative stresses of Patient No. 2 were still greater than those of postoperative Patient No. 4, postoperative symptoms of TMD were different (Fig. $6 \mathrm{~B}$ and $6 \mathrm{D}$ ). It was unavailable for us to obtain the stress magnitude that would lead to TMD. But it's clear that the increased stress postoperatively had more possibility to contribute to the symptoms of TMD.

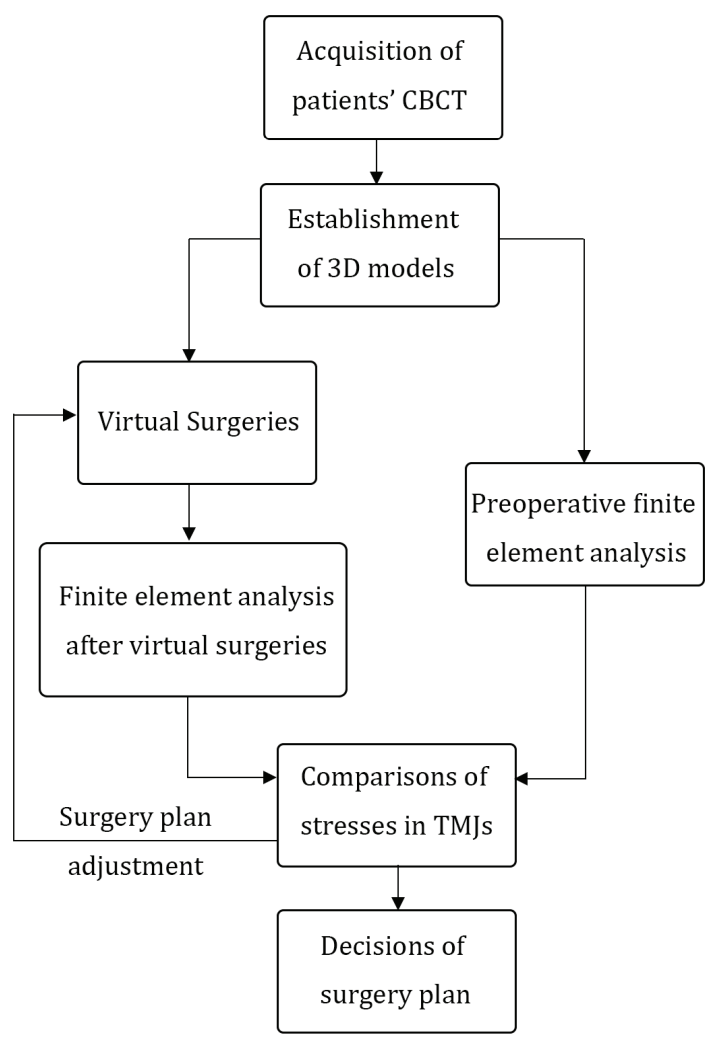

Fig. 7. Future workflow of treatment decisions for surgeons in combination with the biomechanical analysis

The comparison of pre- and postoperative stresses for patients was a feasible way according to virtual surgeries and FEA [20], beneficial for surgeons to adjust operative plans to decrease stresses in TMJs and avoid postoperative TMDs (Fig. 7). In the future, virtual surgeries and finite element analysis could be con- ducted to predict the possible postoperative internal biomechanical environment of TMJs, in order to provide more suitable treatment strategy for mandibular deformity patients. However, there are still some limitations in our study. One major limitation of this study was the muscle forces of individual cannot be detected due to the ethical consideration. Future detection technology may accurately access individual muscle forces, which can be used to verify findings such as ours.

\section{Conclusion}

In summary, effects of BSSRO on stresses in the TMJs of mandibular prognathism patients were generally positive. However, recoveries and occurrence of TMDs after surgeries differed individually. Increased stresses in the TMJs after the surgeries could lead to postoperative symptoms of TMD, while the decreased stress in the TMJs after the surgeries could contribute to recoveries of symptoms accompanied by the mandibular prognathism.

\section{Acknowledgement}

We would like to acknowledge Bingmei Shao and Baiyi Wang from Basic Mechanics Laboratory of Sichuan University, for their assistance and support during the study.

\section{Ethics}

All participants were prospectively provided with IRB (Institutional Review Board) and informed of consent from Stomatological Hospital of Chongqing Medical University.

\section{Funding}

This study was supported by the National Natural Science Foundation of China [Grant Number 31670963] and the Fundamental Research Funds for the Central Universities.

\section{Conflict of interest}

The authors declare no conflict of interest related to this study.

\section{References}

[1] Buranastidporn B., Hisano M., Soma K., Effect of biomechanical disturbance of the temporomandibular joint on the prevalence of internal derangement in mandibular asymmetry, Eur. J. Orthod., 2006, 28, 199-205. 
[2] Cheong H., Lo L., Facial asymmetry: etiology, evaluation and management, Chang Gung Medical Journal, 2011, 34 (4), 341-351.

[3] Da Silva R.J., Valadares Souza C.V., Souza G.A., Ambrosano G.M.B., Freitas D.Q., SANT'ANA E., DE OliveIRA-SAntos C., Changes in condylar volume and joint spaces after orthognathic surgery, Int. J. Oral Maxillofac. Surg., 2018, 47 (4), 511-517.

[4] Del Palomar A.P., Doblare M., Finite element analysis of the temporomandibular joint during lateral excursions of the mandible, J. Biomech., 2006, 39, 2153-2163.

[5] Diernberger S., Bernhardt O., Schwahn C., Kordass B., Self-reported chewing side preference and its associations with occlusal, temporomandibular and prosthodontic factors: results from the population-based Study of Health in Pomerania (SHIP-0), Journal of Oral Rehabilitation, 2008, 35 (8), 613-620.

[6] FAng B., Shen G.F., YAnG C., Wu Y., Feng Y.M., Mao L.X., XIA Y.H., Changes in condylar and joint disc positions after bilateral sagittal split ramus osteotomy for correction of mandibular prognathism, Int. J. Oral Maxillofac. Surg., 2009, 38 (7), 726-730.

[7] Goncalves J.R., Wolford L.M., Cassano D.S., Da Porciuncula G., Paniagua B., Cevidanes L.H., Temporomandibular joint condylar changes following maxillomandibular advancement and articular disc repositioning, J. Oral Maxillofac. Surg., 2013, 71 (10), 1759, e1-15.

[8] Korioth T.W., HANnAm A.G., Mandibular forces during simulated tooth clenching, Journal of Orofacial Pain, 1994, $8(2), 178$.

[9] LIU Z., FAN Y., QIAN Y., Comparative evaluation on three-dimensional finite element models of the temporomandibular joint, Clin. Biomech. (Bristol, Avon), 2008, 23, Suppl. 1, S53-8.

[10] LIU Z., QIAN Y., ZHANG Y., FAN Y., Effects of several temporomandibular disorders on the stress distributions of temporomandibular joint: a finite element analysis, Comput. Methods Biomech. Biomed. Engin., 2016, 19 (2), 137-143.

[11] Liu Z., SHu J., Zhang Y., FAN Y., The Biomechanical Effects of Sagittal Split Ramus Osteotomy on Temporomandibular Joint, Comput. Methods Biomech. Biomed. Engin., 2018, 21 (11), 617-624.

[12] Martinez-Gomis J., Lujan-Climent M., Palau S., Bizar J., SAlSench J., Peraire M., Relationship between chewing side preference and handedness and lateral asymmetry of peripheral factors, Arch. Oral Biol., 2009, 54 (2), 101-107.

[13] Moraes K.J.R.D., Cunha D.A.D., Albuquerque L.C.A., CARValho C.C.D., Silva H.J.D., Chewing preference and its relationship with postural muscular electric potential, Revista CEFAC, 2018, 20 (5), 648-656.

[14] Navarro R.D., Oltramari P.V.P., SAnT'Ana E., Henriques J.F.C., Taga R., Cestari T.M., Conti P.C.R., CunHA F.Q., SANTos C.F., Histological and molecular temporomandibular joint analyses after mandibular advancement surgery: study in minipigs, Oral Surgery Oral Medicine Oral Pathology Oral Radiology and Endodontology, 2008, 106 (3), 331-338.

[15] Prager T.M., Rafayelyan S., Landau H., Pischon N., Minden K., Jost-Brinkmann P.G., Muller-Hartwich R., MUSSLER A., Effect of untreated and treated temporomandibular joint arthritis on mandibular volume development in growing rabbits, J. Orofac. Orthop., 2015, 76 (3), 265-274.
[16] Pruim G.J., De Jongh H.J., Ten Bosch J.J., Forces acting on the mandible during bilateral static bite at different bite force levels, Journal of Biomechanics, 1980, 13 (9), 755-763.

[17] Serel Arslan S., Inal O., Demir N., Olmez M.S., KARADUMAN A.A., Chewing side preference is associated with hemispheric laterality in healthy adults, Somatosens Mot. Res., 2017, 34 (2), 92-95.

[18] SHU J., ZhANG Y., CHONG D.Y.R., LiU Z., Effects on loads in temporomandibular joints for patients with mandibular asymmetry before and after orthognathic surgeries under the unilateral molar clenching, Biomechanics and Modeling in Mechanobiology, 2020, 19, 533-541.

[19] Shu J., Zhang Y., LiU Z., Biomechanical comparison of temporomandibular joints after orthognathic surgery before and after design optimization, Medical Engineering and Physics, 2019, 68, 11-16.

[20] SHU J., Zhang Y., LIU Z., Biomechanical comparison of temporomandibular joints after orthognathic surgery before and after design optimization, Med. Eng. Phys., 2019, 68, 11-16.

[21] Shu J.H., YAO J., Zhang Y.L., ChONG D.Y.R., LIU Z., The influence of bilateral sagittal split ramus osteotomy on the stress distributions in the temporomandibular joints of the patients with facial asymmetry under symmetric occlusions, Medicine (Baltimore), 2018, 97 (25), e11204.

[22] Tanne K., TANaka E., SAKuda M., Stress distribution in the temporomandibular joint produced by orthopedic chincup forces applied in varying directions: A three-dimensional analytic approach with the finite element method, Am. J. Orthod. Dentofacial Orthop., 1996, 110 (5), 502.

[23] TAY D., Physiognomy in the classification of individuals with a lateral preference in mastication, Journal of Orofacial Pain, 1994, 8 (1), 61-72.

[24] Ueki K., Nakagawa K., Takatsuka S., Yamamoto E., The change of stress distribution on the condyle after mandibular setback surgery, Eur. J. Orthod., 2006, 28 (5), 433-439.

[25] Ueki K., Yoshizawa K., Moroi A., Iguchi R., Kosaka A., IKawa H., SaIDA Y., HotTA A., TsuTsuI T., Changes in computed tomography values of mandibular condyle and temporomandibular joint disc position after sagittal split ramus osteotomy, J. Craniofac. Surg., 2015, 43 (7), 1208-1217.

[26] WoLFORD L., The sagittal split ramus osteotomy as the preferred treatment for mandibular prognathism, Journal of Oral and Maxillofacial Surgery, 2000, 58 (3), 310-312.

[27] Wu H., TAo S., The experience of correction of mandibular prognathism by Sagittal split ramus osteotomy, Xian dai kou qiang yi xue za zhi, 1994, 4, 237.

[28] Zhang Y.L., The Effect of Bilateral Sagittal Split Ramus Osteotomy on Temporomandibular Joints in Patients with Facial Asymmetry: a Morphologic and Biomechanical Study, Sichuan University, Chengdu 2017, 42-70.

[29] Zhang Y.L., LiU Y., Shu J.H., Xu X.C., LiU Z., Morphological study of the changes after sagittal split ramus osteotomy in patients with facial asymmetry: measurements of 3-dimensional modelling, Br. J. Oral Maxillofac. Surg., 2018, 56 (10), 925-930.

[30] Zhang Y.L., Song J.L., Xu X.C., Zheng L.L., Wang Q.Y., FAN Y.B., LIU Z., Morphologic Analysis of the Temporomandibular Joint Between Patients With Facial Asymmetry and Asymptomatic Subjects by $2 D$ and $3 D$ Evaluation: A Preliminary Study, Medicine (Baltimore), 2016, 95(13), e3052. 